\title{
2D acoustic cloaks of arbitrary shape with layered structure based on transformation acoustics
}

\author{
Qi Li and Jeffrey S. Vipperman* \\ Department of Mechanical Engineering \& Materials Science, University of Pittsburgh, \\ Pittsburgh, PA 15260, USA
}

\begin{abstract}
Acoustic metamaterials have attracted much attention in recent years. Acoustic cloaks, which make objects invisible to acoustic waves, are the most common use for acoustic metamaterials. In this paper, acoustic cloaks with arbitrary shapes are presented based on transformation acoustics. This method interprets the compression and dilation of space as appropriate properties of materials. The derived properties of the cloak with irregular shapes are highly inhomogeneous and anisotropic, much more complex than the annulus cloaks. The materials for this kind of cloak are impossible to find in nature, and difficult to fabricate with artificial materials. In order to overcome this difficulty, layered structure with isotropic materials is adopted to approximate the required properties of the cloak. Numerical simulations about the cloaks of arbitrary shape are performed to validate the design.
\end{abstract}

Transformation acoustics arose after transformation optics and attracted much attention since then. Transformation acoustics leads to many applications, of which the most intriguing is acoustic cloaking ${ }^{1}$. An acoustic cloak is a device which makes acoustic waves pass around the object it covers and has no impact on the outside acoustic field, as if the object was not there. In 2006, Cummer and Schurig $^{2}$ derived the properties for $2 \mathrm{D}$ cylindrical cloaks. Later on, Chen and $\mathrm{Chan}^{3}$ got the properties of acoustic cloaks in three dimensions. Since perfect cloaking devices are difficult to realize, onedimensional cloaks and ground cloaks are proposed later. For example, Zhu, et al. ${ }^{4}$ presented a design of rhombic cloak with homogeneous metamaterials, which works in one direction and can be used as a ground cloak.

In recent years, many cloaks have been fabricated. In 2011, Zhang, et al ${ }^{5}$ made a 2D cylindrical acoustic cloak for underwater ultrasound based on transmission line approach. $\mathrm{Hu}$, et al. ${ }^{6}$ fabricated the $2 \mathrm{D}$ rhombic acoustic cloak with perforated plates. Zigoneanu, et $\mathrm{al}^{7}$ realized the three-dimensional broadband omnidirectional acoustic ground cloak based on their 2D model in air.

Although much progress has been made in acoustic cloaks, there are still much to do, one of which is to extend them to arbitrary shapes. In electromagnetics, cloaks with arbitrary shapes have been studied. ${ }^{9-11}$ In acoustics, Hu, et al. ${ }^{12}$ and Yang, et al. ${ }^{13}$ studied acoustic cloaks with arbitrary shapes using numerical methods, which validates the feasibility of acoustic cloaks with arbitrary shapes in theory.

In this paper, acoustic cloaks with arbitrary shapes were designed by discretizing the cloak into sections with some radii starting from a specific point within the inner boundary of the cloak. Within each section, transformation acoustics was used to derive the properties of the cloak. Set $\theta$ as constant within each section, the properties were just functions of $r$. Layered structure was used to approximate the anisotropic and inhomogeneous properties of each section. Numerical simulations with COMSOL Multiphysics were performed in the last. This method is more practical which means that acoustic cloaks with arbitrary shapes can be fabricated with homogeneous materials.

When a cloak is put in a homogeneous background, the properties of the cloak are (as in Ref. 1, 3,7)

$$
\begin{gathered}
\frac{\overline{\bar{\rho}}}{\rho_{\mathrm{b}}}=\operatorname{det}(\mathbf{A})\left(\mathbf{A} \mathbf{A}^{\mathrm{T}}\right)^{-1} \\
\frac{\kappa}{\kappa_{b}}=\operatorname{det}(\mathbf{A})
\end{gathered}
$$

where $a_{i j}=\frac{\partial x_{i}{ }^{\prime}}{\partial x_{j}} \cdot x_{i}{ }^{\prime}$ is the coordinate in the cloak and $x_{j}$ is 
the coordinate in the original space. $\rho_{b}$ is the mass density of the background, and $\kappa_{b}$ is the bulk modulus of the background. $\overline{\bar{\rho}}$ and $\kappa$ are the density tensor and bulk modulus of the cloak.

For 2D cloaks of arbitrary shape in polar coordinate

$$
\mathbf{A}=\left[\begin{array}{cc}
\frac{\partial r^{\prime}}{\partial r} & \frac{\partial r^{\prime}}{r \partial \theta} \\
\frac{r^{\prime} \partial \theta^{\prime}}{\partial r} & \frac{r^{\prime} \partial \theta^{\prime}}{r \partial \theta}
\end{array}\right]
$$

where, $r$ and $\theta$ are coordinates in virtual space, and $r^{\prime}$ and $\theta^{\prime}$ are coordinates in physical space.

The derived bulk modulus is inhomogeneous, while the density is anisotropic and inhomogeneous. What's more, the density tensor is non-diagonal, which makes it more difficult.

To make it easier, we let

$$
\left\{\begin{array}{l}
r^{\prime}=R_{\text {in }}(\theta)+\frac{R_{\text {ex }}(\theta)-R_{\text {in }}(\theta)}{R_{\text {ex }}(\theta)} r \\
\theta^{\prime}=\theta
\end{array}\right.
$$

where, $R_{\text {in }}(\theta)$ is the function of the interior boundary of the cloak, and $R_{e x}(\theta)$ is the function of the exterior boundary of the cloak.

The properties of the cloak can be obtained as

$$
\begin{aligned}
\frac{\overline{\bar{\rho}}}{\rho_{b}} & =\left[\begin{array}{cc}
\frac{r}{r-R_{\text {in }}} & -\frac{a_{12}}{1-\frac{R_{\text {in }}}{R_{\text {ex }}}} \\
-\frac{a_{12}}{1-\frac{R_{\text {in }}}{R_{\text {ex }}}} & \frac{r-R_{\text {in }}}{r}\left(1+\frac{a_{12}{ }^{2}}{\left(1-\frac{R_{\text {in }}}{R_{\text {ex }}}\right)^{2}}\right)
\end{array}\right] \\
\frac{\kappa}{\kappa_{b}} & =\left(1-\frac{R_{\text {in }}}{R_{\text {ex }}}\right)^{2} \frac{r}{r-R_{\text {in }}}
\end{aligned}
$$

where,

$$
a_{12}=\frac{1-\frac{R_{\text {in }}}{R_{e x}}}{r-R_{\text {in }}} \frac{\partial R_{\text {in }}}{\partial \theta}-\frac{\partial}{\partial \theta}\left(\frac{R_{\text {in }}}{R_{\text {ex }}}\right)
$$

The derived density and bulk modulus are functions of $\theta$ and $r$. However, the whole domain can be divided into several sections. Then within each section, $\theta$ can be approximated as constant. Then, the properties are just functions of $r$. When the sections are small enough, the approximation is accurate.

Although the properties within each section are independent of $\theta$ due to our approximation, the properties are still anisotropic and inhomogeneous. What's more, the mass density has off-diagonal elements. Since the density tensor is symmetric, it can be transformed to the principal axes where the mass matrix is diagonal. The transformation matrices vary with each section, and are calculated numerically for each.

The derived diagonal density tensors are still anisotropic, but can be approximated by the layered structure, as in Ref 13, 14. When the thickness of each layer is much smaller than the wavelength, the alternating layer structure can be described as an anisotropic medium. With two homogeneous and isotropic layers A (mass density $\rho_{\mathrm{A}}$, bulk modulus $\kappa_{\mathrm{A}}$ ) and B (mass density $\rho_{\mathrm{B}}$, bulk modulus $\kappa_{\mathrm{B}}$ ) arranged alternatively, the effective densities and bulk modulus are

$$
\begin{gathered}
\rho_{r}=\frac{\rho_{\mathrm{A}}+\eta \rho_{\mathrm{B}}}{1+\eta} \\
\frac{1}{\rho_{\theta}}=\frac{1}{1+\eta}\left(\frac{1}{\rho_{\mathrm{A}}}+\frac{\eta}{\rho_{\mathrm{B}}}\right) \\
\frac{1}{\kappa}=\frac{1}{1+\eta}\left(\frac{1}{\kappa_{\mathrm{A}}}+\frac{\eta}{\kappa_{\mathrm{B}}}\right)
\end{gathered}
$$

where $\eta=d_{\mathrm{B}} / d_{\mathrm{A}}$ is the ratio of thicknesses for $\mathrm{B}$ and $\mathrm{A}$ layers.

For a square cloak, as shown in Figure 1 (left), the boundaries are lines. Since the properties of the four sections are the same, we can just calculate the section in the first quadrant. The interior and exterior boundaries in the first quadrant can be expressed as

$$
\begin{aligned}
& R_{\text {in }}(\theta)=\frac{a}{\cos (\theta)+\sin \theta} \\
& R_{\text {ex }}(\theta)=\frac{b}{\cos (\theta)+\sin \theta}
\end{aligned}
$$

in polar coordinates.

Substitute equation (6) into equation (4), we can get the density and bulk modulus of the cloak. As mentioned above, the properties are functions of both $\theta$ and $r$. However, as shown in Figure 1 (right), the cloak can be divided into $N$ small sections, within each of which $\theta$ can be seen as constant, denoted as $\theta_{i}=\left(i-\frac{1}{2}\right) \frac{2 \pi}{N},(i=1,2, \cdots$

$, N)$.

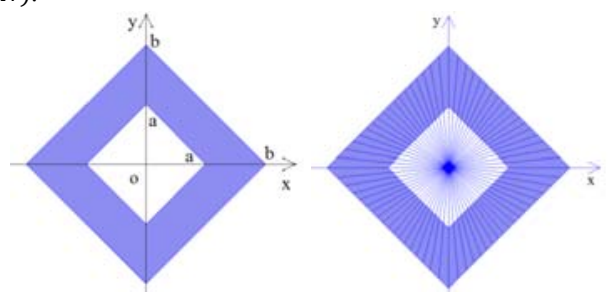

FIG.1 Configuration (left) and division (right) of the square cloak
Commented [J V1]: Potentially confusing because you have " $\mathrm{a}_{\mathrm{ij}}$ " in equation (4) 
Then the density tensor becomes a function of $r$ only, but has off-diagonal elements. Fortunately, the matrix is symmetric. It can be diagonalized by an orthogonal matrix. That is,

$$
\boldsymbol{\Lambda}=\mathbf{Q}^{T} \frac{\overline{\bar{\rho}}}{\rho_{b}} \mathbf{Q}
$$

where, $\mathbf{Q}$ is an unitary matrix, the columns of which are eigenvectors of $\overline{\bar{\rho}} / \rho_{b}$; the elements of the diagonal matrix $\boldsymbol{\Lambda}$ are the corresponding eigenvalues. $\mathbf{Q}$ shows the direction of the principal axes relative to the original coordinate, and the elements of $\boldsymbol{\Lambda}$ are the relative densities in principal directions. $\boldsymbol{\Lambda}$ can be expressed as

$$
\boldsymbol{\Lambda}=\left[\begin{array}{cc}
\rho_{r} & 0 \\
0 & \rho_{\theta}
\end{array}\right]
$$

The transformed properties can be approximated by layered structures perpendicular to the radial principal direction with equation (5). The properties of the layered structures are

$$
\begin{gathered}
\rho_{\mathrm{A}}, \rho_{\mathrm{B}}=\left(\rho_{r} \pm \sqrt{\rho_{r}^{2}-\rho_{r} \rho_{\theta}}\right) \times \rho_{b} \\
\kappa_{\mathrm{A}}, \kappa_{\mathrm{B}}=\kappa
\end{gathered}
$$

With the properties and principal directions calculated, the total pressure field with and without the cloak are computed with COMSOL Multiphysics finite element analysis (FEA) software. The results in two different directions are shown in Figure.2. From the results we can see that the waves pass around the object as if no obstacle was there.

For a elliptical cloak, each section after division is not a quadrilateral because of the nonlinear boundary. A piecewise linear boundary is adopted as approximation to calculate the properties. The simulation results of total pressure field in COMSOL Multiphysics are shown in Figure 3.

From the simulation results we can see that the cloaks reduce the reflections and shadows due to an object in the acoustic field, rendering the object almost undetectable in acoustic field. The cloaks function well no matter in which direction the plane wave propagates. Therefore, these cloaks are functional and omnidirectional.

The condition for layered structure to function as an effective anisotropic material is that the thickness of each layer is much smaller than the wavelength. The simulation of the elliptical cloak for plane waves with different frequencies is shown in Figure.4. The cloak works well within certain frequencies. So these cloaks are broadband. Since at lower frequencies, the wavelengths are larger. These cloaks work better at lower frequencies.

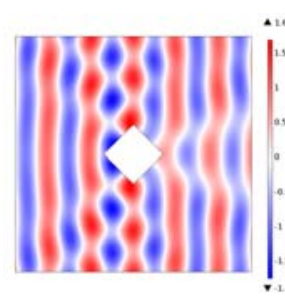

(a)

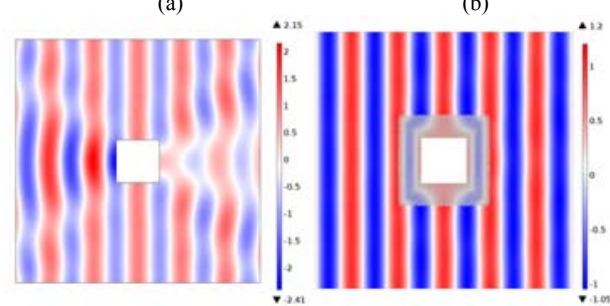

(c)

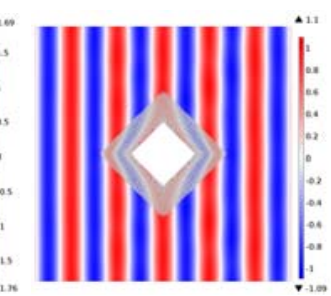

(b)
FIG. 2 Simulations for a square cloak. (a) pressure field without cloak in direction 1. (b) pressure field with square cloak in direction 1. (c) pressure field without cloak in direction 2. (d) pressure field with square cloak in direction 2.

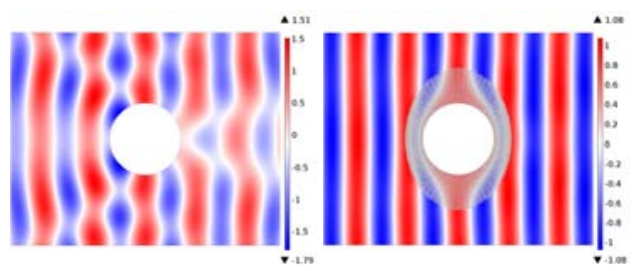

(a)

(b)

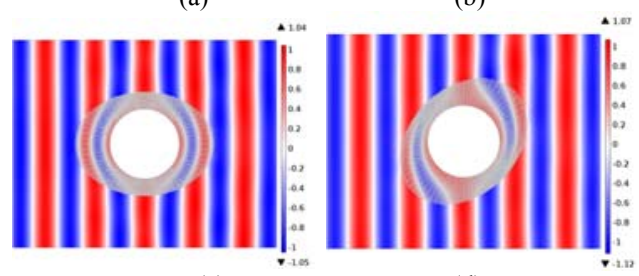

(c)

FIG. 3 Simulations for elliptical cloak. (a) pressure field without cloak (b) pressure field with elliptical cloak in direction 1. (c) pressure field with elliptical cloak in direction 2. (d) pressure field with elliptical cloak in direction 3. 


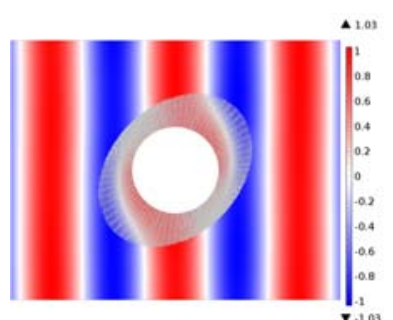

(a)

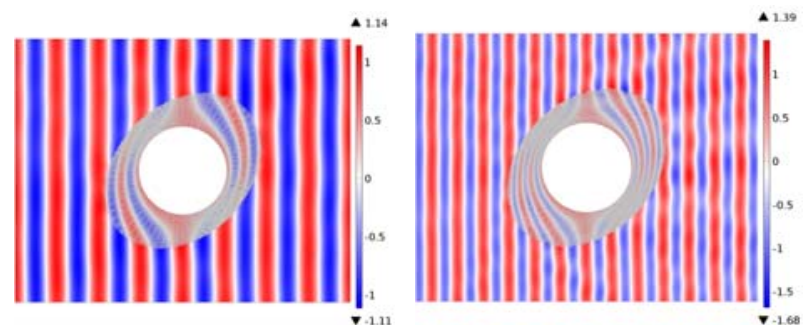

(b)

(c)

FIG.4 simulation for waves with different frequencies (a) $f_{1}$ (b) $3 f_{1}$ (c) $5 f_{1}$

Like the annulus cloak $^{13}$, the densities of these cloaks become extreme near the inner boundaries. The relative properties for different sections of elliptical cloak are shown in Figure 5 and Figure 6. The properties of the cloak make it difficult to realize in current practice, but offers the opportunity for further exploration.

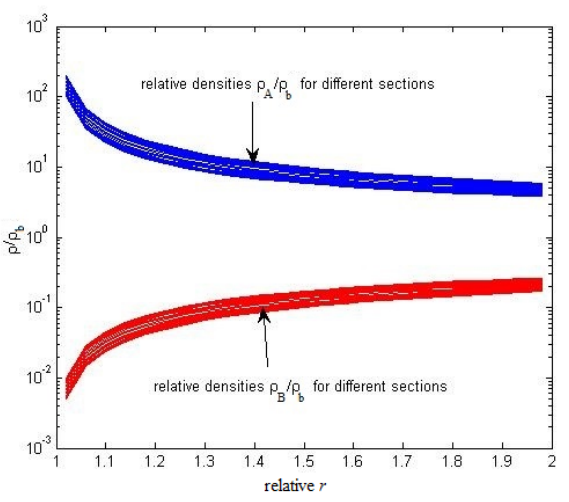

FIG. 5 Relative densities for different sections

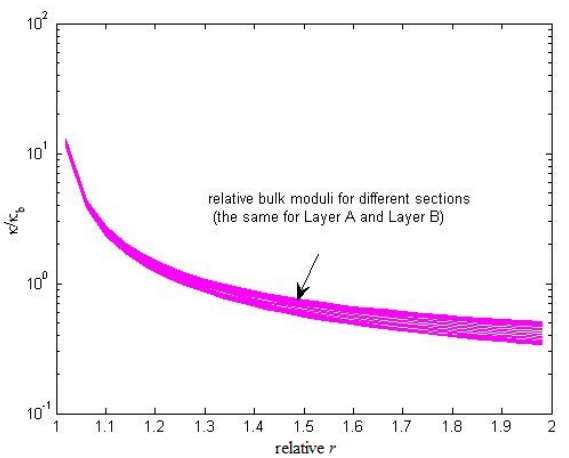

FIG. 6 Relative bulk moduli for different sections

In conclusion, cloaks with arbitrary shapes can be achieved by discretizing the cloak into small sections and using layered structures in each section. The cloaks designed with this method are broadband and omnidirectional when the size of each domain is much smaller than the wavelength.

\section{Reference}

${ }^{1}$ H. Chen and C. T. Chan, J. Phys. D: Appl. Phys. 43, 113001 (2010). Doi: 10.1088/0022-3727/43/11/113001

${ }^{2}$ Steven A Cummer and David Schurig, New J. Phys, 9, 45 (2007). Doi: $10.1088 / 1367-2630 / 9 / 3 / 045$

${ }^{3}$ H. Chen and C. T. Chan, Appl. Phys. Lett. 91, 183518 (2007). Doi: 10.1063/1.2803315

${ }^{4}$ W. Zhu, C. Ding, and X. Zhao, Appl. Phys. Lett. 97, 131902 (2010). Doi: $10.1063 / 1.3492851$

${ }^{5}$ S. Zhang, C. Xia, and N. Fang, Phys. Rev. Lett. 106, 024301 (2011). Doi: 10.1103/PhysRevLett.106.024301

${ }^{6}$ W. Hu1, Y. Fan, P. Ji and J. Yang, J. Appl. Phys. 113, 024911 (2013). Doi: 10.1063/1.4775408

${ }^{7}$ L. Zigoneanu, B. Popa and S. A. Cummer, Nature Materials, 13, 352-355 (2014). Doi: 10.1038/nmat3901

${ }^{8}$ B.-I. Popa, L. Zigoneanu, and S. A. Cummer, Phys. Rev. Lett. 106, 253901(2011). Doi: 10.1103/PhysRevLett.106.253901

${ }^{9}$ Q.Wu, K. Zhang, F.Meng and L. Li, J. Phys. D: Appl. Phys. 42, 035408 (2009). Doi: 10.1088/0022-3727/42/3/035408

${ }^{10}$ J. Zhang, Y. Luo, H. Chen, and B. Wu, J. Opt. Soc. Am. B 25, 11 (2008). Doi: 10.1364/JOSAB.25.001776

${ }^{11}$ W. X. Jiang, J. Y. Chin, Z. Li, Q. Cheng, R. Liu, and T. J. Cui, Phys. Rev. E 77, 066607 (2008). Doi: 10.1103/PhysRevE.77.066607

${ }^{12}$ J. Hu, X. Zhou, G. Hu, Comput. Mater. Sci. (2009). doi:10.1016/j.commatsci.2009.02.006

${ }^{13}$ J. J. Yang, M. Huang, G. H. Cai, R. S. Xie, J. Yang, J. Vib. Acoust. 135(5), 051024 (2013). doi: 10.1115/1.4024559

${ }^{14}$ Y. Cheng, F. Yang, J. Y. Xu, and X. J. Liu, Appl. Phys. Lett. 92, 151913 (2008). Doi: 10.1063/1.2903500

${ }^{15}$ T. Daniel, and J. Sánchez-Dehesa, New J. Phys., 10.6 (2008): 063015. doi:10.1088/1367-2630/10/6/063015 